\title{
Collaboration of Intelligent Interoperable Agents Via Smart Interface
}

\author{
Evgeniy Bryndin \\ Research Department, Research Center "Natural Informatics," Novosibirsk, Russia
}

Email address:

bryndin15@yandex.ru

To cite this article:

Evgeniy Bryndin. Collaboration of Intelligent Interoperable Agents Via Smart Interface. International Journal on Data Science and Technology. Vol. 5, No. 4, 2019, pp. 66-72. doi: 10.11648/j.ijdst.20190504.11

Received: December 7, 2019; Accepted: December 20, 2019; Published: December 31, 2019

\begin{abstract}
Artificial intelligence is a revolutionary technology that is designed to transform the life of the world community: to optimize business processes, to provide valuable information, to increase creative service to citizens. The importance of integrating artificial intelligence into the infrastructure of the future has already been recognized. The Government AI Readiness Index has been created, which reflects the readiness of Governments to support the development of artificial intelligence technology. The coming years will take to improve security and standardize the development and use of intelligent agents that ensure their compatibility. Intelligent agents can be combined at the software level through a standard interface to communicate with them based on mental real mathematics. Compatibility will allow produce from them intelligent ensembles with cognitive creative and behavioral abilities of the person for service services. It will also allow produce intelligent production high-tech complexes. Standardizing the cooperation of intelligent agents will help to ensure the interface, compatibility and synergy of their safe application in various sectors of economy, industry and service. Creative ensembles of intelligent interoperable agents, which implement technological, production, service, commercial, research and other creative processes, are an incentive for a breakthrough in the field of artificial intelligence for the sustainable development of society. In the future, creative ensembles of intellectual interoperable agents will qualitatively change the life of the world community.
\end{abstract}

Keywords: Artificial Intelligence, Intelligent Interoperable Agents, Smart Interface, Creative Processes, Creative Ensemble

\section{Introduction}

Intelligent agents have a well-developed and complementary substantive model of the outside world, which is achieved by having a knowledge base, decision mechanisms and analysis of actions. The intelligent agent, based on the essence model of the external environment, is able to make his own judgements and on their basis make independent decisions, perform actions that change the environment [1-19]. The difference between types of intelligent agents is related to functions. In communicative agents, the internal model of the world turns mainly into a model of communication consisting of models of participants, the process and the desired result of communication.

A full intellectual agent must have four of these functions: cognitive, regulatory, communicative, and resource. They are characterized by appropriate behavior in the agent community, as well as a desire to use the resources of other agents to achieve their own goals. They are endowed with their own mechanisms of motivation, which give rise to the goals that determine their actions. Full intellectual cognitive agents, due to their complexity, knowledge and ability to judge their behavior and external environment, are autonomous. They work relatively independently, demonstrating quite flexible behavior. But the ability to resist external influences causes some difficulties in organizing their effective interaction. In the ensemble, which includes only intellectual agents, there are a limited number of them.

Humanoid agents associated with internal models of other agents and capable of reflexion are characterized by social role behavior. The complexity of the behavior depends on the functions and program of the agent. Agent functions are defined by a mathematical description, and an agent program is a specific implementation that operates within agent architecture.

Intelligent agents have the following properties: 
1. Autonomy - the ability to function without interference and to monitor the internal state and its actions;

2. Social behaviour - possibility of interaction and communication with other agents;

3. Reactivity - adequate perception of the medium and appropriate reactions to its changes;

4. Activity - the ability to act in a rational way to achieve the goal;

5. Basic knowledge - knowledge of the agent about itself, the environment, other agents that do not change within the life cycle;

6. Variable part of the basic knowledge that may change over time, although the agent may not be aware of it and may continue to use it for his own purposes;

7. Targets - a set of states to which the current behavior of the agent is aimed;

8. Obligations - the tasks undertaken by the agent at the request of other agents;

9. Intent is what the agent must do by virtue of its obligations.

Hybrid architectures of intelligent agent ensembles are used to solve real-world problems. In the hybrid architecture of a professional ensemble, the collaboration of intelligent agents develops on the basis of big data attributes.

\section{Data Attributes}

Objects, objects, materials, things, processes, phenomena and other aspects of the physical world have different properties.

Properties are represented by qualitative content attributes.

Qualitative attribute can be visual or audible. A meaningful attribute can be represented by a number, a language sense value, a visual or audible way, a mathematical or behavioral action, or an algorithm.

Meaningful qualitative attributes are artificial intelligence data.

Big Data - the data collected automatically is too large for manual collection and processing to find new patterns and knowledge that cannot be derived from local data fragments.

Big Smart Data (Big SD). A collection of qualitative and quantitative attributes related in time, space, and subject area.

Big SD attributes of the fields of economics, industrial industries, technology and professions help build and train artificial neural multilayer artificial intelligence networks to manage, make decisions and issue recommendations to professionals and executives.

Applied research modeling helps accumulate Big SD scientific attributes in real time and simultaneously use them to deeply train multi-layer artificial neural control networks to simulate applied research, make decisions, and make recommendations to researchers.

Big data attributes are used in the process of intelligent agent collaboration through the standard interface of their interaction.

\section{Intelligent Agent Collaboration Via Smart Interface}

In modern agent - oriented technologies and methods of building agent ensembles an important place is the study of interaction (communication) of agents, and the greatest difficulty is the support of interaction of intelligent agents. Despite the abundance of work in this field, there are practically no descriptions of research related to the construction of sufficiently universal models of interaction of intelligent agents. There is a Muller model where the dialogue model is considered as a set of simplest interaction protocols, with each protocol being defined in terms of the states of the interaction participants and the actions of the participants causing transitions between these states, which allows to describe the protocols of interaction of the participants in the form of a non-deterministic state machine. There is a common model of intelligent agent interaction [1]. The disadvantage of such an approach is that the experience of all interactions of intelligent agents is ignored and retaught to improve the quality of cooperation.

Such a task is solved by a smart interface and mental real mathematics.

Mental real mathematics is based on the sustainability of physical and spiritual laws, positive natural numbers and Big SD. Real mathematics brings natural and humanities together and makes artificial intelligence more natural. Intelligent agents are trained on Big SD of completed processes, facts, events. And they give the necessary results on possible big intelligent data (Big SD) in the process of their interaction.

Smart interface stores all intelligent agent interactions, a large array of data available for analysis is accumulated. For each interface member that needs to enter or select something, can take values from this data array. A smart interface can find patterns in the behavior of intelligent agents and make decisions based on them. A smart interface can understand how it has been used before, analyze repeats, find the reasons for these repeats and ways to interpret scenarios from the past to improve the user experience now. He notices repetitive actions, independently collects and analyzes statistics, and identifies patterns and new patterns. By identifying patterns, embeds learning elements into the interface, and retraining accumulates experience.

When collecting a story, the smart interface learns what data and functions intelligent agents use. If the agent ignores an important and convenient function, the interface can analyze the history of its activities and train it if necessary. By correcting and complementing the functions that the interface builds, the intelligent agent will improve the quality of interaction.

Intelligent interface providing direct interaction of intelligent agents in task solving, performs functions:

a) Enabling the user to set the solution program;

b) Provision of forms of information presentation;

c) Providing flexible interaction of intelligent agents;

d) Program execution support;

e) Replenishing the knowledge and data base; 
f) Providing retraining of intelligent agents.

The interaction of intelligent agents is carried out on the basis of the presentation of knowledge and data of the subject area of the task and the intelligent interface. Developing the abilities and capabilities of a comfortable and high-quality interaction of a person with a system that organizes and provides this, and is a smart intellectual interface. The intelligent interface is managed by a software smart manager. Intelligent agents themselves prepare interaction data in the correct format. Interface sends them to the appropriate agent.

The smart interface combines the hybrid intelligence of human-machine agents for social and production practices. Artificial intelligence of machine agents is realized by cognitive components, such as advisory and prognostic systems, biometric complexes, knowledge management, natural virtual dialogue, machine learning with reinforcement, convolutional neural networks, neural chips, and others [4-7]. Complexes of intelligent interoperable agents that implement technological, production, service, commercial, research and other creative processes form creative ensembles.

\section{Creative Ensembles of Intelligent Interoperable Agents}

Creative ensembles of intelligent interoperable agents are the focus of distributed artificial intelligence of multiagent systems. The technology of multi-agent systems, although it has more than a decade of active development, is still in the process of being developed. Active research is under way in the field of theoretical foundations of formalization of basic concepts and components of systems.

The current section of the article discusses a creative ensemble for solving one problem by several intelligent interoperable agents. The task is divided into several subtasks, which are distributed among the agents. Another area of consideration is to provide interaction between agents when one agent may generate a request to another agent to transmit some data or perform certain actions. And also ensure the ability to transfer knowledge. For example, social processes in which each of the agents performs its function in a subject area represented as a set of agents, then independent tasks can be performed by different agents when control and responsibility for the actions performed are distributed among the agents.

Agents in creative ensembles are parts of a single system and solve subtasks of one common task. However, the agent cannot work outside the system. It is believed that one agent has only a partial view of the global problem, which means that he can solve only some part of the overall problem. Therefore, in order to solve the difficult task, it is necessary to create some set of agents and organize effective interaction between them, which will allow build a single creative ensemble of intelligent interoperable agents. The efficient interaction of intelligent interoperable agents provides a smart interface.

Creative ensemble is a complex of intelligent interoperable agents interacting through a smart interface, implementing either technological process, social services, multi-inter- transdisciplinary research, or production cycle. The scheme of creative ensemble with smart interface is shown in Figure 1.

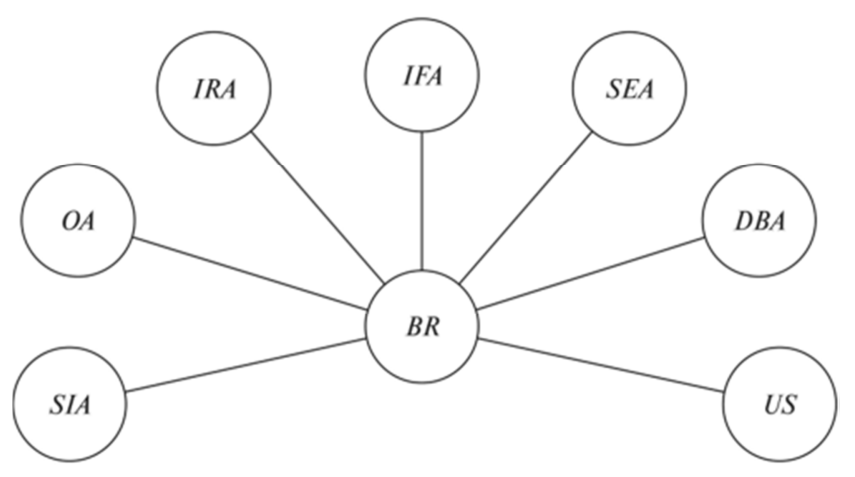

Figure 1. Creative Ensemble Scheme with Smart Interface.

In the creative ensemble, the whole range of tasks by certain rules is distributed among all agents. Job allocation means assigning each agent a role whose complexity is determined by the agent 's capabilities.

To organize the task distribution process, the creative ensemble creates either a distributed problem solution system or decentralized artificial intelligence. In the first version, the process of decomposition of the global problem and the inverse process of composition of the found solutions takes place under the control of some single "center." At the same time, the creative ensemble is designed strictly from top to bottom, based on the roles defined for the agents and the results of dividing the global task into subtasks. In the case of decentralized artificial intelligence, task distribution occurs during agent interaction and is synergistic. Synergy of creative ensembles with man is formed in the direction of cognitive robotics of production and social sphere [8-19].

The use of a creative ensemble of intelligent interoperable agents is described in Case 1.

Case 1. Application of creative ensemble

Intelligent interoperable agents

Table 1. General.

\begin{tabular}{ll}
\hline Use case name & Application of creative ensemble of intelligent interoperable agents \\
\hline Application domain & Hi-Tech Labor Market \\
Deployment Model & Human digital double \\
Status & Results of research: Strong Artificial Distributed Intelligence \\
Scope & Economic sectors and social services \\
Objective (s) & Find accurate and universal application of strong artificial distributed intelligence \\
& Short $\quad$ Creative ensemble is complex of intelligent interoperable agents interacting through smart interface, \\
Narrative & description (not implementing either technological process, social services, multi-inter- trans-disciplinary research, or \\
& more than 150 production cycle. \\
\hline
\end{tabular}




\begin{tabular}{|c|c|c|c|c|}
\hline Use case name & \multicolumn{4}{|c|}{ Application of creative ensemble of intelligent interoperable agents } \\
\hline \multirow{5}{*}{$\begin{array}{l}\text { Stakeholders } \\
\text { Stakeholders' assets, values } \\
\text { System's threats and } \\
\text { vulnerabilities }\end{array}$} & $\begin{array}{l}\text { Complete } \\
\text { description }\end{array}$ & \multicolumn{3}{|c|}{$\begin{array}{l}\text { Creative ensemble is complex of intelligent interoperable agents interacting through smart interface, } \\
\text { implementing either technological process, social services, multi-inter- trans-disciplinary research, or } \\
\text { production cycle. In the creative ensemble, the whole range of tasks by certain rules is distributed } \\
\text { among all agents. Job allocation means assigning each agent a role whose complexity is determined by } \\
\text { the agent's capabilities. To organize the task distribution process, the creative ensemble creates either a } \\
\text { distributed problem solution system or decentralized artificial intelligence. In the first version, the } \\
\text { process of decomposition of the global problem and the inverse process of composition of the found } \\
\text { solutions takes place under the control of some single "center." At the same time, the creative } \\
\text { ensemble is designed strictly from top to bottom, based on the roles defined for the agents and the } \\
\text { results of dividing the global task into subtasks. In the case of decentralized artificial intelligence, task } \\
\text { distribution occurs during agent interaction and is synergistic. }\end{array}$} \\
\hline & \multicolumn{4}{|c|}{ Highly technological producer } \\
\hline & \multicolumn{4}{|l|}{ Reputation } \\
\hline & \multicolumn{4}{|c|}{ Legal and ethical aspects of interaction with society. } \\
\hline & ID & Name & Description & $\begin{array}{l}\text { Reference to } \\
\text { mentioned use case } \\
\text { objectives }\end{array}$ \\
\hline \multirow[t]{2}{*}{$\begin{array}{l}\text { Key performance indicators } \\
\text { (KPIs) }\end{array}$} & 1 & $\begin{array}{l}\text { AI management } \\
\text { of professional } \\
\text { cooperation } \\
\text { process }\end{array}$ & $\begin{array}{l}\text { The technology of creative processes control can itself } \\
\text { predict optimal terms of execution of certain stages on the } \\
\text { basis of accumulated information about their labour } \\
\text { intensity, selection of the route of staff load and } \\
\text { competences of employees. Optimize processes during } \\
\text { their execution - automatic delegation of tasks taking into } \\
\text { account the load of employees and their competences. }\end{array}$ & Improve accuracy \\
\hline & 2 & $\begin{array}{l}\text { Productivity } \\
\text { and quality AI }\end{array}$ & $\begin{array}{l}\text { Strong artificial intelligence works with fewer mistakes } \\
\text { and is safer. Strong artificial improves the quality of life } \\
\text { of man and society in daily concerns, as well as } \\
\text { productivity in high-tech industry and production. }\end{array}$ & \multirow[t]{5}{*}{ Improve efficiency } \\
\hline \multirow{4}{*}{ AI features } & \multirow{4}{*}{$\begin{array}{l}\text { Task (s) } \\
\text { Method (s) } \\
\text { Hardware } \\
\text { Topology } \\
\text { Terms and } \\
\text { concepts used }\end{array}$} & \multicolumn{2}{|l|}{ Creative activity } & \\
\hline & & \multicolumn{2}{|l|}{ Deep learning } & \\
\hline & & \multicolumn{2}{|c|}{$\begin{array}{l}\text { Supercomputer with Strong Artificial Distributed Intelligence } \\
\text { Distributed Modular Interconnect Topology }\end{array}$} & \\
\hline & & \multicolumn{2}{|c|}{ Deep learning, "imagification", neural network, training, training data set } & \\
\hline \multirow{3}{*}{$\begin{array}{l}\text { Standardization } \\
\text { opportunities/requirements } \\
\text { Challenges and issues }\end{array}$} & \multicolumn{4}{|c|}{ Strong artificial distributed intelligence requires process standardization, as does every human activity. } \\
\hline & \multicolumn{4}{|c|}{ Qualitatively new type of thinking not available to humans. } \\
\hline & \multirow{2}{*}{$\begin{array}{l}\text { Description } \\
\text { SDGs to be } \\
\text { achieved }\end{array}$} & \multicolumn{3}{|c|}{ Security and ethical and legal aspects } \\
\hline Societal concerns & & \multicolumn{3}{|c|}{ Universal approach to big data processing with smart cognitive systems } \\
\hline
\end{tabular}

Table 2. Data.

\begin{tabular}{ll}
\hline Data characteristics & \\
\hline Description & Strong Artificial Distributed Intelligence Data \\
Source & Model and technology of Strong Artificial Distributed Intelligence \\
Type & Strong \\
Volume (size) & Hi-Tech Labor Market \\
Velocity (e.g. real time) & Supercomputering Velocity \\
Variety (multiple datasets) & streams of multiple datasets \\
Variability (rate of change) & Retraining \\
Quality & High \\
\hline
\end{tabular}

Table 3. Process scenario.

\begin{tabular}{|c|c|c|c|c|c|}
\hline \multicolumn{6}{|c|}{ Scenario conditions } \\
\hline N. & Scenario name & Scenario description & Triggering event & Pre-condition & Post-condition \\
\hline 1 & Training & $\begin{array}{l}\text { Train a model (deep neural } \\
\text { network) with training data set }\end{array}$ & $\begin{array}{l}\text { Technological process raw data } \\
\text { set is ready }\end{array}$ & Formatting of data & Management of safety \\
\hline 2 & Evaluation & Expansion of the trained model & $\begin{array}{l}\text { Development of technological } \\
\text { thinking and behaviour }\end{array}$ & $\begin{array}{l}\text { Cognitive thinking patterns } \\
\text { and psychological } \\
\text { behaviors }\end{array}$ & $\begin{array}{l}\text { Meeting KPI } \\
\text { requirements is condition } \\
\text { of development }\end{array}$ \\
\hline 3 & Execution & Model and Technology Tooling & Interaction & Activization of Model & $\begin{array}{l}\text { Completion of } \\
\text { interaction }\end{array}$ \\
\hline 4 & Retraining & Retrain a model with training & Certain period of time has passed & Additional data and & Combining Data and \\
\hline
\end{tabular}




\begin{tabular}{llllll}
\hline \multicolumn{2}{l}{ Scenario conditions } & & & & \\
\hline N. & Scenario name & Scenario description & Triggering event & Pre-condition & Post-condition \\
\hline & data set & since the last training/retraining & knowledge & Knowledge \\
\hline
\end{tabular}

Table 4. Training.

\begin{tabular}{|c|c|c|c|c|c|}
\hline Scenario name & Training & & & & \\
\hline Step No. & Event & Name of process/Activity & Primary actor & Description of process/activity & Requirement \\
\hline 1 & $\begin{array}{l}\text { Sample raw } \\
\text { data set is ready }\end{array}$ & $\begin{array}{l}\text { Specification and } \\
\text { classification }\end{array}$ & Manufacturer & Transform sample raw data & $\begin{array}{l}\text { Distributed AI } \\
\text { Software }\end{array}$ \\
\hline 2 & $\begin{array}{l}\text { Completion of } \\
\text { Step } 1\end{array}$ & $\begin{array}{l}\text { Creating Set of } \\
\text { Experimental Data }\end{array}$ & Manufacturer & $\begin{array}{l}\text { Development of set of experimental } \\
\text { data through job modelling }\end{array}$ & Software of modelling \\
\hline 3 & $\begin{array}{l}\text { Completion of } \\
\text { Step } 2\end{array}$ & Model training & $\begin{array}{l}\text { AI solution } \\
\text { provider }\end{array}$ & $\begin{array}{l}\text { Train a model (deep neural } \\
\text { network) with experimental data set } \\
\text { created by Step } 2\end{array}$ & Big SD \\
\hline
\end{tabular}

Table 5. Evaluation.

\begin{tabular}{llllll}
\hline Scenario name & Evaluation & & & & \\
\hline Step No. & Event & Name of process/Activity & Primary actor & Description of process/activity & Requirement \\
\hline 1 & $\begin{array}{l}\text { Completion of } \\
\text { training/retraining }\end{array}$ & Research & Manufacturer & $\begin{array}{l}\text { Train model (deep neural } \\
\text { network) with experimental data } \\
\text { set created }\end{array}$ & Big SD \\
Based on data, detect execution & \\
2 & $\begin{array}{l}\text { Completion of } \\
\text { Step 1 }\end{array}$ & Identification & AI solution provider & $\begin{array}{l}\text { using a deep neural network } \\
\text { trained in learning scenario }\end{array}$ & Big SD \\
Completion of & Evaluation & Manufacturer & Compan of phase 2 results & Efficiency and \\
with human performance & quality \\
$\begin{array}{l}\text { Input of evaluation } \\
\text { Output of evaluation }\end{array}$ & Productivity & & & \\
\hline
\end{tabular}

Table 6. Execution.

\begin{tabular}{|c|c|c|c|c|c|}
\hline Scenario name & Execution & & & & \\
\hline Step No. & Event & Name of process/Activity & Primary actor & Description of process/activity & Requirement \\
\hline 1 & $\begin{array}{l}\text { Completion of } \\
\text { comparison of modeling } \\
\text { results with human } \\
\text { performance }\end{array}$ & Research & Manufacturer & $\begin{array}{l}\text { Development of a set of } \\
\text { experimental data through job } \\
\text { modelling }\end{array}$ & Quality \\
\hline 2 & Completion of Step 1 & Identification & Manufacturer & $\begin{array}{l}\text { Based on modified data train } \\
\text { model (deep neural network) with } \\
\text { experimental data set created }\end{array}$ & Compatibility \\
\hline \multicolumn{2}{|c|}{ Input of Execution } & Modification & & & \\
\hline \multicolumn{2}{|c|}{ Output of Execution } & Compatibility & & & \\
\hline
\end{tabular}

Table 7. Retraining.

\begin{tabular}{|c|c|c|c|c|c|}
\hline Scenario name & Retraining & & & & \\
\hline Step No. & Event & Name of process/Activity & Primary actor & Description of process/activity & Requirement \\
\hline 1 & $\begin{array}{l}\text { Certain period of time } \\
\text { has passed since the last } \\
\text { training/retraining }\end{array}$ & Research & Manufacturer & Additional data and knowledge & Completeness \\
\hline 2 & Completion of Step 1 & Experimental data set creation & Manufacturer & $\begin{array}{l}\text { Combining Data and Knowledge } \\
\text { Based on modified data train } \\
\text { model (deep neural network) with } \\
\text { experimental data set created }\end{array}$ & Compatibility \\
\hline 3 & Completion of Step 2 & Model training & $\begin{array}{l}\text { AI solution } \\
\text { provider }\end{array}$ & $\begin{array}{l}\text { Comparison of phase } 2 \text { results } \\
\text { with human performance }\end{array}$ & $\begin{array}{l}\text { Efficiency } \\
\text { and quality }\end{array}$ \\
\hline \multicolumn{2}{|c|}{ Specification of retraining data } & \multicolumn{4}{|c|}{ Retraining data set has to include recent data } \\
\hline
\end{tabular}

\subsection{Practical Application of Creative Ensemble of Intelligent Interoperable Agents}

\subsubsection{Virtual Enterprise}

The creation of virtual enterprises is one of the modern lines of business, which is greatly stimulated by the rapid growth of information resources and services provided on the
Internet. In addition, the emergence of virtual enterprises is facilitated by the reduction of the life cycle time of the created products and the improvement of their level of complexity, as this creates the necessity of operational integration of production, technological and intellectual resources. Another important reason is the tightening of competition in commodity markets, which styles the 
association of enterprises for survival purposes. A virtual enterprise can be defined as a co-operation of legally independent enterprises, organizations and dividends that produce products or services in a common business process. In the outside world, a virtual enterprise acts as a single organization that uses management and administration systems based on the adoption of information and telecommunication technologies. The purpose of creating a virtual enterprise is to combine production, technological, intellectual and investment resources to market new products and services. Since each real enterprise, within a virtual enterprise, performs only part of the work from the overall technological chain, two main tasks are solved during its creation. This is the decomposition of the overall business process into components (sub-processes). The second task is to choose the economic composition of the real partner enterprises that will carry out the technological process. The first task is solved using methods of system analysis, and multi-agent technologies are used to solve the problem. The task of optimally distributing a plurality of jobs (processes) among a plurality of workers (real enterprises) in the operations study is formulated as a task about values.

\subsubsection{Multi-agent Decision Support System}

Intelligent multi-agent decision-making systems are designed to assess the quality of organizational, technical and economic decisions in the process of the enterprise. At present, there is a shift from the concept of one hundred business to mobile business, in which the concepts of competitiveness and flexibility play a major role. In order to operate in new rapidly changing conditions, enterprises need to constantly transform their production structures and business process structures. At the same time, it becomes inevitable to attract third-party specialists in the field of technology, marketing, reengineering, etc. The evaluation of the proposed solutions is a complex and permanent activity involving the participation of highly qualified experts from different fields of knowledge, which are usually geographically separated from each other. This is due to the relevance of distributed computer support of decision-making processes in enterprises, which can be implemented with the application of multi-agent systems. The general decisionmaking scheme includes the following steps: specification of requirements; generation of solutions; assessment of alternatives; choosing an effective solution. The evaluation of decisions is carried out by a working group consisting of a manager, an analyst and experts. The functions among the team participants are distributed as follows. The manager forms a set of indicators (criteria) that will be used to evaluate projects (decisions), composition of the expert group, compose personal document according to which experts perform their own work. Each expert works according to the individual scenario proposed by the manager. The analyst, whose functions can be performed by the manager, expresses his opinion on the results of the work carried out by the experts. To support the group decision-making process, the software implementation of the hierarchy analysis method is used, where the following main procedures are implemented:

a) Formation and coordination of hierarchical structure of indicators;

b) Evaluation and harmonization of quality indicators of the project;

c) Evaluation and harmonization of the importance of indicators;

d) Ranking of alternative solutions and harmonization of results.

Many experts are involved in solving the above-mentioned problems, so at each stage there are procedures for communicating their opinions. Two-level harmonization mechanism is used to coordinate the work of the expert team. Each of the experts is represented by an agent whose task is to evaluate the alternatives offered by the manager according to a given set of quality indicators. With the help of the knowledge editor, the manager provides tasks to experts and analyses the information received from them. The task of coordinating agent behaviour is entrusted to the coordinating agent. The results of the system are agreed expert evaluations, on the basis of which multi-criterion ranking of alternatives is carried out.

\subsubsection{Multi-agent Technologies for Solving Complex Problems}

Multi-agent technologies are based on a fundamentally new method of solving complex problems, which are not solved or difficult to solve by classical mathematical methods. In multi-agent technologies, the solution of the problem is obtained in the course of self-organization of many software agents capable of competition and cooperation, and having their own criteria, preferences and restrictions. A solution is considered found when, in the course of their undeterministic interactions, agents reach the best consensus (balance of interests) that is taken as the solution to the task. The solution of the problem is considered as a balance when no agent can improve his condition any more, which indicates the achievement of a reasonable compromise, balance of interests or agreement (harmony) of all participants in solving the problem situation.

\subsubsection{Electronic Multi-agent Store}

Its main purpose is to organize interaction between buyer agents and store agents whose interests coincide. An electronic multi-agent store is a community of electronic store agents (sellers, managers, cashiers, security) and buyers who can communicate and exchange information through electronic means of communication in the complete (or minimal) absence of personal direct contact. A virtual store agent is a Web server for selling goods and services to other Internet users. It must provide the customer with information about the item (service) and receive an order for the item (service) from the customer. The cashier agent receives payment when using online payment systems. The agent manager checks out the shipment of the paid item if the buyer does not pick up the item himself. The robot agentconsultant greets customers in the store, helps them to find a specific product on the whole sales window, answers general 
questions. The electronic agent guard through the review system tracks the buyers of the store. In the event of a breach, he blocks the door until the police who serve the store arrive.

\section{Conclusion}

The coming years will take to improve safety and standardize the development and application of a viable creative ensemble of intelligent interoperable agents. International standardization of the production and use of intelligent agents ensuring their compatibility has intensified. Intelligent agents can be combined at the software level through a smart interface to communicate with them. Compatibility will allow produce creative ensembles from them with cognitive creative and behavioral abilities of the person for service services and production processes. It will also allow produce intelligent production high-tech complexes. Standardization of viable creative ensembles of intelligent interoperable agents will help to ensure the interface, compatibility and synergy of their safe application in various sectors of economy, industry and service. Developing approaches to creating creative artificial intelligence ensembles that will be beneficial to humanity remains one of the most pressing challenges. Mental real mathematics will be an incentive for the development of intellectual interoperable agents. In order to create ensembles of intelligent interoperable agents, it is necessary to train specialists Big SD in natural and human sciences. Already currently experts in mental mathematics show abilities to work with Big SD. In the future, reliable and safe viable creative ensembles of intelligent interoperable agents will qualitatively change the life of the world community in the interests of sustainable development [20].

\section{References}

[1] Petukhov. Intelligent agent interactions. Collection of scientific works of the international conference - KnowledgeDialogue-Solution (KDS '2001), S. - Pb: ACKIC, 2001.

[2] Melihova O. A., Gregory A. S., Jmbinov SV, Chumichev V. S., Gaidukov A. B. Methods of training in artificial intelligence systems. Compilation of the LII International Scientific Practical Conference. ANS Sibak, 2015 - P. 19-29.

[3] Evgeniy Bryndin. System retraining to professional competences of cognitive robots on basis of communicative associative logic of technological thinking. International Robotics Automation Journal. 2019; 5 (3): 112-119.

[4] Evgeniy G. Bryndin. Modeling of Transformation of Nanostructures by Cognitive Systems on the Basis of Big Smart Data. International Journal of Artificial Intelligence and Mechatronics. Volume 7, Issue 4. 2019. P. 19-22.

[5] Evgeniy Bryndin Formation Smart Data Science for Automated Analytics of Modeling of Scientific Experiments.
American Journal of Software Engineering and Applications. Volume 8, Issue 2, 2019. Pages: 36-43.

[6] Evgeniy Bryndin. Mainstreaming technological development of industrial production based on artificial intelligence. COJ Technical \& Scientific Research, 2 (3). 2019. Pages: 1-5.

[7] Evgeniy Bryndin. Practical Development of Creative LifeSaving Strong Artificial Intelligence. Communications, Vol. 7, No. 1, 2019. Pages.

[8] Evgeniy Bryndin. Cognitive Robots with Imitative Thinking for Digital Libraries, Banks, Universities and Smart Factories. International Journal of Management and Fuzzy Systems. V. 3 , N. 5, 2017, pp 57-66.

[9] Evgeniy Bryndin. Technological Thinking, Communication and Behavior of Androids. Communications. Vol. 6, No. 1, 2018. Pages: 13-19.

[10] Venture radar - Top Service Robotics Companies, 2018. URL:

https://www.ventureradar.com/keyword/Service\%20Robotics.

[11] Pedro Domingos. Supreme algorithm. How machine learning will change our world. - M.: Mann, Ivanov and Ferber, 2016. - 336 pages

[12] Evgeniy Bryndin. Social Cognitive Smart Robots: Guide, Seller, Lecturer, Vacuum Cleaner, Nurse, Volunteer, Security Guard, Administrator. Communications. Volume 7, Issue 1. 2019. Pages: 6-12.

[13] Evgeniy Bryndin. Directions of Development of Industry 4.0, Digital Technology and Social Economy. American Journal of Information Science and Technology. Volume 2, Issue 1. 2018 P. 9-17.

[14] Evgeniy Bryndin. Practical Development of Creative LifeSaving Strong Artificial Intelligence. Communications, 7, Issue 2, 2019. Pages: 31-39.

[15] Evgeniy Bryndin. Human Digital Doubles with Technological Cognitive Thinking and Adaptive Behaviour. Software Engineering, Volume 7, Issue 1, 2019. P. 1-9.

[16] Artificial Intelligence \& Robotics: Industry Report \& Investment Case. 2019 https://indexes.nasdaqomx.com/docs/NQROBO\%20Research. pdf.

[17] Deep Learning based Recommender System: A Survey and New Perspectives, Shuai Zhang et al., 2018. https://arxiv.org/pdf/1707.07435.pdf.

[18] Google's Artificial Intelligence Built an AI That Outperforms Any Made by Humans. 2019 https://futur-ism.com/googleartificial-intelligence-built-ai.

[19] Evgeniy Bryndin. Robots with Artificial Intelligence and Spectroscopic Sight in Hi-Tech Labor Market. International Journal of Systems Science and Applied Mathematic, V. 4, № 3, 2019. Pages: 31-37.

[20] Evgeniy Bryndin. Creative innovative transformational ecosystem of formation of humane technological society. International Robotics Automation Journal. 2019; 5 (3): 91-94 